\title{
MicroRNAs as markers of progression in cervical cancer: a systematic review
}

\author{
Barbara Pardini ${ }^{1,2}$, Daniela De Maria ${ }^{1}$, Antonio Francavilla' ${ }^{1}$, Cornelia Di Gaetano ${ }^{1,2}$, Guglielmo Ronco ${ }^{3+}$ \\ and Alessio Naccarati ${ }^{1 *+}$ (D)
}

\begin{abstract}
Background: Invasive cervical cancer (ICC) is caused by high-risk human papillomavirus types (HR-HPVs) and is usually preceded by a long phase of intraepithelial neoplasia (CIN). Before invasion, (epi) genetic changes, potentially applicable as molecular markers within cervical screening, occur in HPV host cells. Epigenetic alterations, such as dysregulation of microRNA (miRNA) expression, are frequently observed in ICC. The mechanisms and role of miRNA dysregulation in cervical carcinogenesis are still largely unknown.

Methods: We provide an overview of the studies investigating miRNA expression in relation to ICC progression, highlighting their common outcomes and their weaknesses/strengths. To achieve this, we systematically searched through Pubmed database all articles between January 2010 and December 2017.

Results: From the 24 studies retrieved, miR-29a and miR-21 are the most frequently down- and up-regulated in ICC progression, respectively. Microarray-based studies show a small overlap, with miR-10a, miR-20b, miR-9, miR-16 and miR106 found repeatedly dysregulated. miR-34a, miR-125 and miR-375 were also found dysregulated in cervical exfoliated cells in relation to cancer progression.

Conclusions: The pivotal role of miRNAs in ICC progression and initial development is becoming more and more relevant. Available studies are essentially based on convenience material, entailing possible selection bias, and frequently of small size: all these points still represent a limitation to a wide comprehension of miRNAs relevant for ICC. The targeted approach instead of a genome-wide investigation still precludes the identification of all the relevant miRNAs in the process. The implementation of deep sequencing on large scale population-based studies will help to discover and validate the relation between altered miRNA expression and CC progression for the identification of biomarkers. Optimally, once explored on a miRNome scale, small specific miRNA signatures maybe used in the context of screening.
\end{abstract}

Keywords: Cervical cancer, Cancer progression, microRNA, Cervical intraepithelial neoplasia (CIN) lesions, HPV infection, Microarray, qPCR

\section{Summary}

Altered microRNA expression is observed in cervical cancer and precancerous lesions. Reviewing the literature, miR-29a and miR-21 are frequently dysregulated in cancer progression. However, microarray-based studies show a small overlap, most studies are based on convenience material and frequently small.

\footnotetext{
* Correspondence: alessio.naccarati@iigm.it

${ }^{\dagger}$ Guglielmo Ronco and Alessio Naccarati contributed equally to this work. ${ }^{1}$ Italian Institute for Genomic Medicine (IIGM), Via Nizza 52, 10126 Turin, Italy Full list of author information is available at the end of the article
}

\section{Background}

Invasive cervical cancer (ICC) is the fourth most common cancer in women worldwide with an estimated 528,000 new cases and 266,000 deaths in 2012 [1]. Infection by $\mathrm{Hu}-$ man Papillomavirus (HPV) is a necessary cause of ICC [2] but the large majority of infections clear spontaneously [3]. Persistent infections can result in intraepithelial lesions, commonly histologically classified as cervical intraepithelial neoplasia (CIN) grade 1 to 3 . About $1 / 3$ of CIN3 progress to invasion in 30 years [4]. CIN1 is considered as a morphologic expression of HPV infection and CIN2 as a mixture of CIN1 and CIN3, frequently regressing [5]. The

(c) The Author(s). 2018 Open Access This article is distributed under the terms of the Creative Commons Attribution 4.0 International License (http://creativecommons.org/licenses/by/4.0/), which permits unrestricted use, distribution, and reproduction in any medium, provided you give appropriate credit to the original author(s) and the source, provide a link to the Creative Commons license, and indicate if changes were made. The Creative Commons Public Domain Dedication waiver (http://creativecommons.org/publicdomain/zero/1.0/) applies to the data made available in this article, unless otherwise stated. 
mechanisms of carcinogenesis are only partly understood. Progression to intraepithelial pre-cancers involves the disruption of cell cycle control pathways mediated by overexpression of the viral E6 and E7 proteins that, among other actions, functionally inactivate the products of the RB and p53 immuno-suppressive genes. Progression to invasion involves the accumulation of genetic errors. The overall process is complex and includes a number of genetic and epigenetic alterations [6, 7].

Screening based on testing for HPV has been shown to be more effective than cytology-based ones and to allow longer screening intervals [8]. However, because of what described above, the positive predictive value of HPV testing for high-grade CIN is low, requiring further triage. Cytological triage is effective [8] but entails high frequency of colposcopy and test repeats [9]. Markers allowing to improve this process would be very useful, so as markers allowing to reduce the overtreatment of non-progressive CIN2/3.

miRNAs are short non-coding RNAs that modulate gene expression either by catalyzing mRNA cleavage or by inhibiting mRNA translation [10]. The mature miRNA is a single stranded $\sim 22$ nucleotide RNA, sequentially processed from a primary transcript (pri-miRNA) and the resulting stem-loop structure (pre-miRNA) by the Drosha and Dicer proteins, respectively [11]. miRNAs are epigenetic regulators. Many miRNAs are tissue- or differentiation-specific and their temporal expressions modulate gene expression by pairing with complementary nucleotide sequences of the target mRNAs [12]. miRNAs may be overexpressed or down regulated in cancers [13] and have been associated with genetic (e.g. deletions, amplifications and point mutations) and epigenetic (histone modifications and aberrant DNA methylation) alterations [14, 15]. Human miRNAs are frequently located at fragile sites and chromosomal regions affected in cancer. Therefore chromosomal alterations are thought to represent a major mechanism underlying altered miRNA expression in cancer, as already demonstrated in melanoma, neuroblastoma, myeloma cell lines and for ovarian and breast cancer [16].

Evidence from cell lines and (pre) malignant lesions supports the involvement of miRNAs at every stage of ICC development [17-21]. However, little is still known about the specific miRNAs involved and the mechanisms behind their deregulation [22]. Aberrant miRNA expression seems to result from HR-HPV infection [18]. Some miRNA loci localize to fragile sites, where HR-HPV DNA integration may occur. Proteins encoded by HR-HPV can influence host miRNAs expression. HR-HPV E6 and E7 proteins modulate the expression of DNA methyltransferases, enzymes regulating gene expression by methylating their promoter regions [22, 23].

The aim of this review is to comprehensively evaluate the published literature focused on miRNA profiling in relation to progression to ICC. We are aware that some of the studies hereby reported have been recently reviewed by others [24-26]. However, we have updated the studies and we have provided additional information not previously covered. In particular, we tried to identify common miRNA profiles (up- or down-regulated miRNAs) that occur during the progression from normal cervical epithelium, via different CIN lesions, to SCCs in primary tissue or in cervical scraping. The downstream effect on target genes of the identified miRNA signatures and relevant pathways emerging are also briefly explored. Finally, we also provide an overview on the experimental approaches so far used to analyze miRNA expression and the design of the studies, including information on available HPV genotyping.

\section{Methods}

\section{Literature search}

We selected all studies focused on the dysregulation of miRNA expression during progression of cervical carcinogenesis, especially in intraepithelial lesions. Between January 2010 and December 2017 we systematically searched PubMed for publications (non-review) in English initially with "miRNA/microRNA" and "cervical cancer" as keywords, then supplemented by "progression", "CIN", "pre-cancerous lesions", "cervical exfoliated cells". Besides this search, we searched literature in PubMed by using MeSH terms (Cervical Neoplasms and MicroRNAs) with the following approach: "Uterine Cervical Neoplasms" [MeSH Terms] AND ("microRNAs" [MeSH Terms] OR "microRNAs" [All Fields] OR "microRNA" [All Fields]) AND ("microRNAs" [MeSH Terms] OR "microRNAs" [All Fields] OR "miRNAs" [All Fields]) AND ("microRNAs" [MeSH Terms] OR "microRNAs" [All Fields] OR ("micro" [All Fields] AND "RNA" [All Fields]) OR "micro RNA" [All Fields]) AND ("microRNAs" [MeSH Terms] OR "microRNAs" [All Fields] OR "miRNA" [All Fields]) AND ("2006/ 01/01" [PDAT]: "2017/12/31" [PDAT]) AND Journal Article [ptyp] NOT Review [ptyp]. Two independent persons investigated the literature retrieved (A.N, D.D.M.).

Several studies were excluded according to the following criteria (one was sufficient for exclusion): (1) non-cervical cancers considered; (2) miRNAs searched in serum or plasma; (3) miRNAs studied only in cellular lines or in normal versus tumor tissue, without relation to progression from healthy to CIN lesions to SCC; (4) miRNAs investigated only in advanced disease, lymph node metastasis or in relation to prognosis or treatment, radiation and chemotherapy, or (5) studies based on functional experiments, miRNA-related polymorphisms, miRNA target genes, HPV or miRNA methylation, or reporting only computational approaches. 
Twenty four studies were identified and included in the present review (See workflow of selection in Additional file 1: Figure S1).

For each study: author (s), publication year, number and characteristics of patients, analyzed tissue, HPV status and profiling platform and direction (up or down regulation) of differentially expressed miRNAs among different lesions were recorded. The PRISMA statement was followed for systematic reviews http://prisma-statement.org/PRISMAStatement/PRISMAStatement.aspx.

\section{miRNA target genes, gene enrichment and pathway analysis}

For miRNAs reported as dysregulated in two or more studies (separately up- or down-regulated), we searched for validated target genes using the miRWalk 2.0 database (http:// zmf.umm.uni-heidelberg.de/apps/zmf/mirwalk2) [27]. In the majority of situations the $-3 p$ or $-5 p$ miRNA arms was not specified in the retrieved studies, so we have arbitrarily selected one of them (if present) by an additional literature search on the most common arms explored in the cervical cancer field, or in cancer in general. Of all the retrieved validated target genes, we excluded those overlapping between up- and down-regulated miRNAs. Resulting lists of target genes were tested using the Enrichr software (http://amp. pharm.mssm.edu/Enrichr/), for their over-representation in biological pathways. Enrichr is an integrative web-based software application that includes gene-set libraries, available for analysis and download [28]. In particular, for the present research we investigated: KEGG (http://www.genome.jp/ kegg/), and Virus Mint (http://amp.pharm.mssm.edu/Harmonizome/resource/Virus+MINT). The relevance of each gene set enrichment was assessed by a $p$-value adjusted for multiple testing based on the hypergeometric distribution. Gene sets with probability $<5 \%$ were considered as significantly overrepresented.

The final list of genes was also investigated in the Cervical Cancer Gene Database (CCDB) [29] which reports separately up- or down-regulated genes involved in ICC.

\section{Results}

An overview of the 24 identified studies is reported in Table 1. Notably, some of them investigated the overall progression from normal epithelium to ICC, while others only focused on intraepithelial lesions. The techniques used most frequently to evaluate miRNA expression were i) quantitative real -time PCR (qPCR, 18 articles), based on candidate miRNA (s) [30-47], and ii) microarray, either by manufacturer or customized (five articles), [16, 48-51]. Only one study analyzed miRNA expression qualitatively by reverse-transcriptase PCR (RT-PCR) [52]. Array studies widely differed for the number of miRNAs investigated (from 202 to 875). In candidate-miRNA studies, authors analyzed only one or few miRNAs based on literature data or their own previous studies. A few studies performed a discovery phase (generally by arrays) with subsequent validation by a different technique (usually qPCR) [47, 49, 50], as recommended, for example, by the Minimum Information for Publication of Quantitative Real-Time PCR Experiments (MIQE) guidelines [53].

In general, a great variability between studies can be observed, both as for the number of investigated subjects/ samples (12 to 1021, most coming from convenience material without specification of selection methods) and the type of samples. Most studies analyzed formalin-fixed, paraffin-embedded tissue (FFPE) or frozen (stored in RNA later or PBS) cervical tissues. Only three studies used cervical exfoliated cells [44-46]. All specimens were collected at enrollment, before any treatment. Several studies reported HPV genotyping data or at least a stratification of samples in HPV-negative and positive but a few did not report any information.

The studies based on microarrays are first described and, within each, results are compared with those of 'validation' studies by qPCR conducted on the same miRNAs by the same or by other authors.

The first microarray study was published by Pereira and colleagues in 2010 [48]. Authors reported data obtained by an array spotted in house on 281 human miRNAs in 25 independent biological samples. Authors report high variability of miRNA expression, especially among normal samples and could not identify miRNAs significantly upor down regulated in pre- or malignant vs. normal samples. In order to minimize such variability, authors prepared a pool of normal samples. With such approach they identified 21 miRNAs with statistically significant differential expression between the pool of normal samples, a group of CIN1 and CIN3 and the SCC samples. The expression of 8 of such miRNAs (miR-26a, miR-29a, miR-143, miR-145, miR-99a, miR-199a, miR-203, and miR-513) progressively decreased and that of $5 \mathrm{miR}$ NAs (miR-10a, miR-132, miR-148a, miR-196a, andmiR-302b) progressively increased in these three groups. Conversely, there was a decrease moving from normal tissue to CIN followed by an increase from CIN to SCC for six miRNAs (miR-16, miR-27a, miR-106a, miR-142-5p, miR-197, and miR-205) and an increase followed by a decrease in two (miR-522* (now miR-522-5p) and miR-512-3p). No validation of the whole results was reported.

A subsequent array study was performed by Li Y and colleagues on 18 tissue samples, including HPV16-positive SSCs and CIN [49]. Out of 875 tested miRNAs, 31 (14 down-regulated and 17 up-regulated) showed significant trends from normal epithelium to cancer. Six of them (miR-29a, miR-92a, miR-99a, miR-155, miR-195, and miR-375) were validated and confirmed by qPCR in 91 biopsies (24 SCCs and 24 CIN2/3, 43 normal tissue). In 


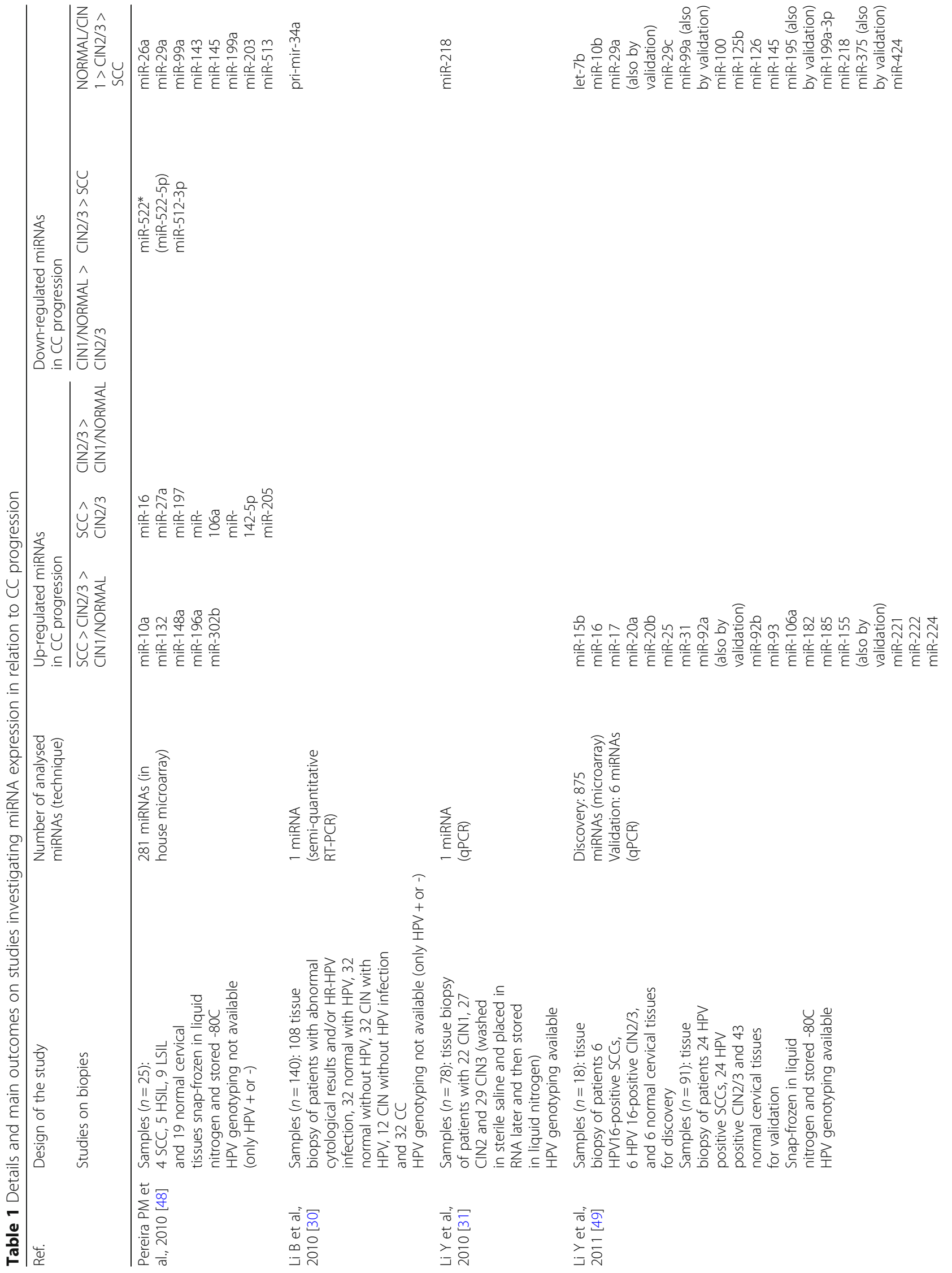




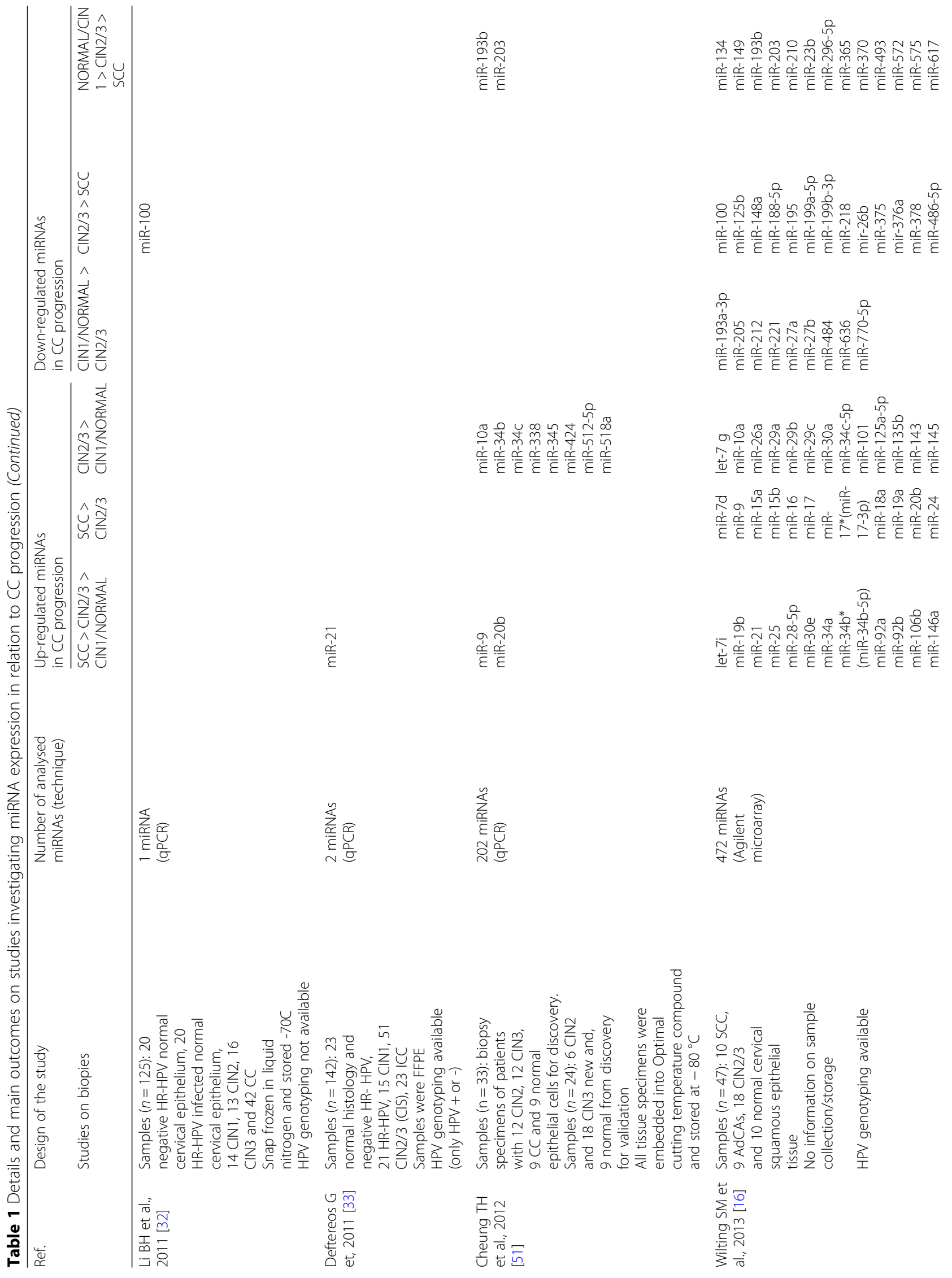




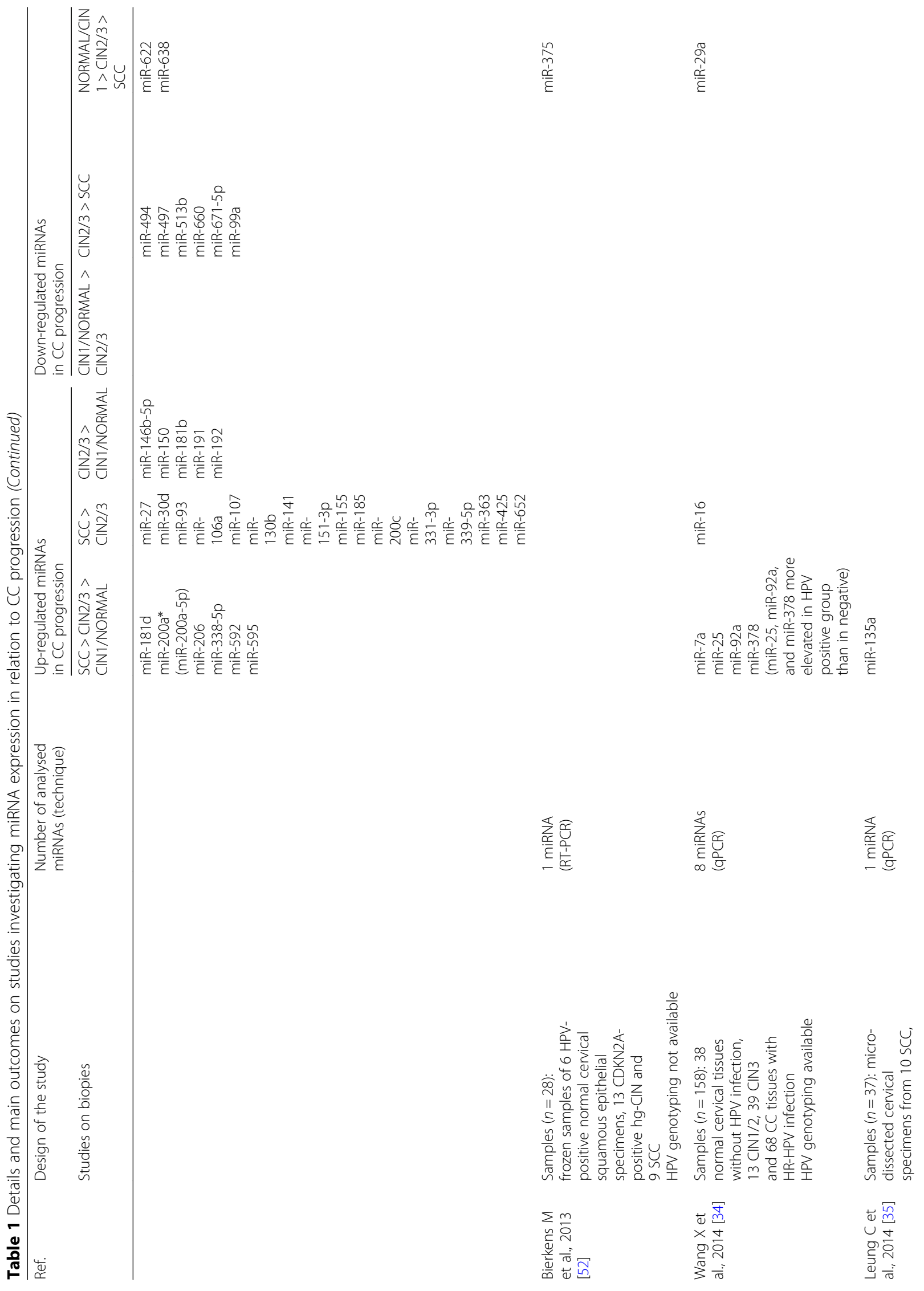




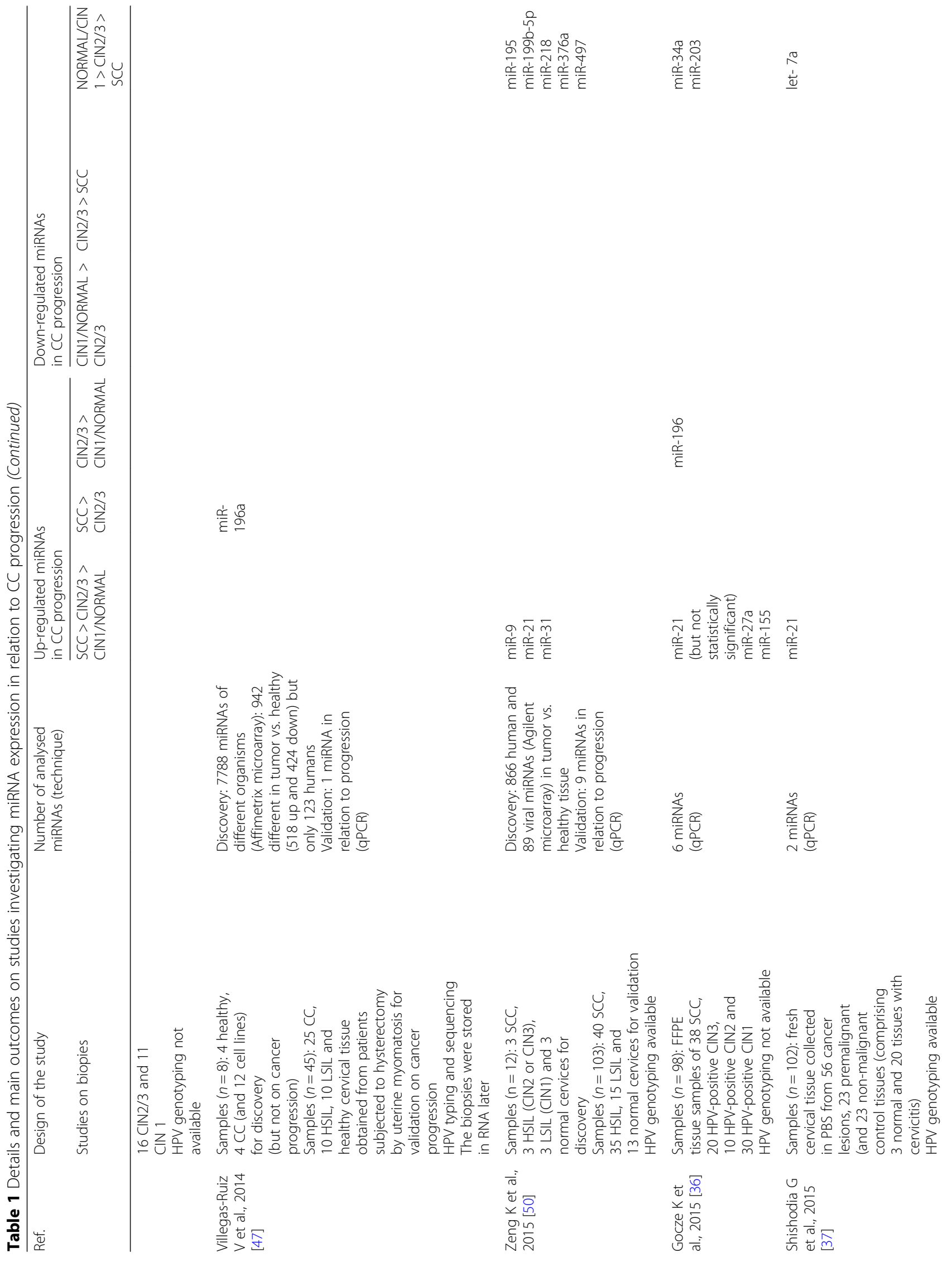




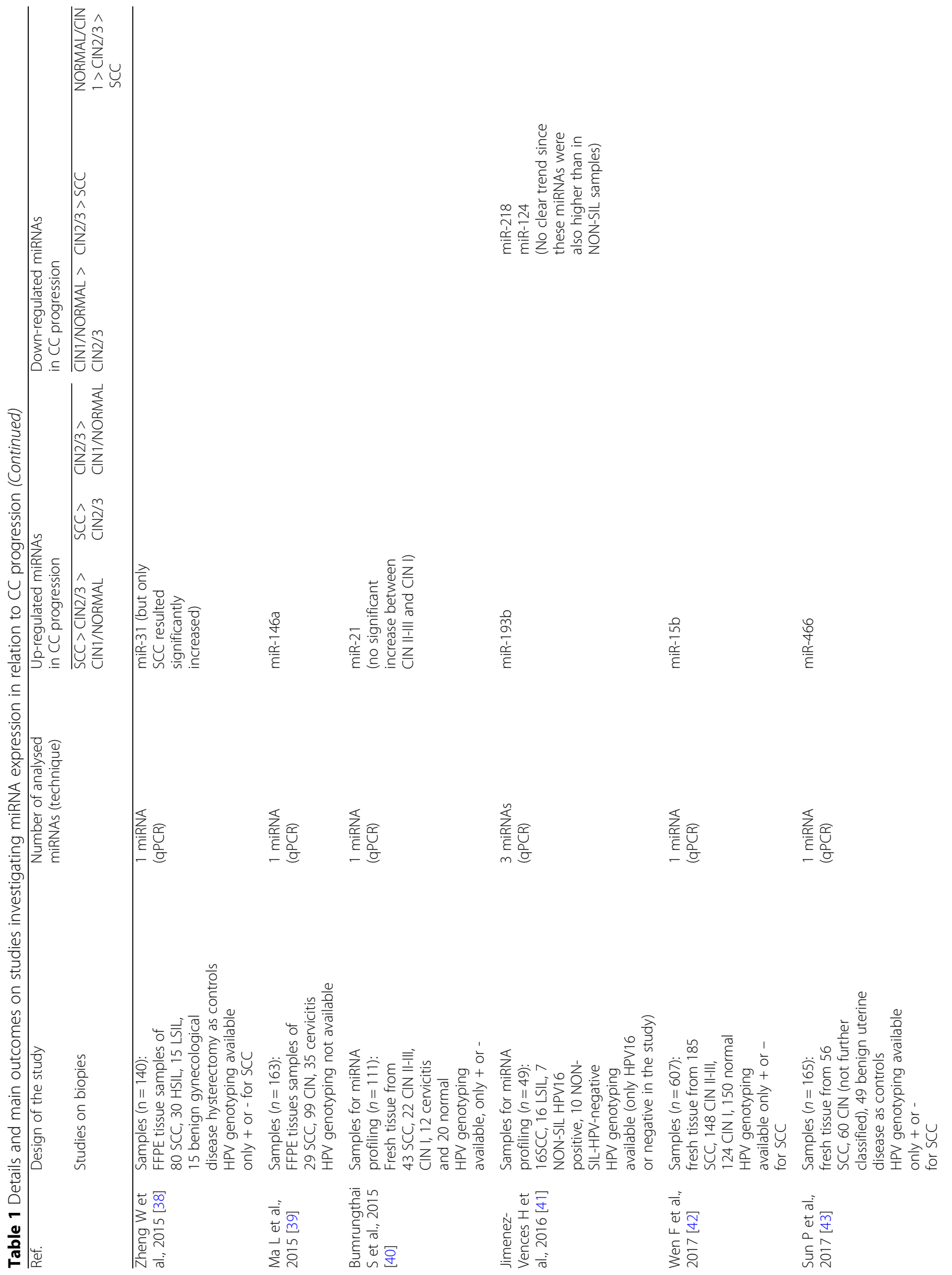




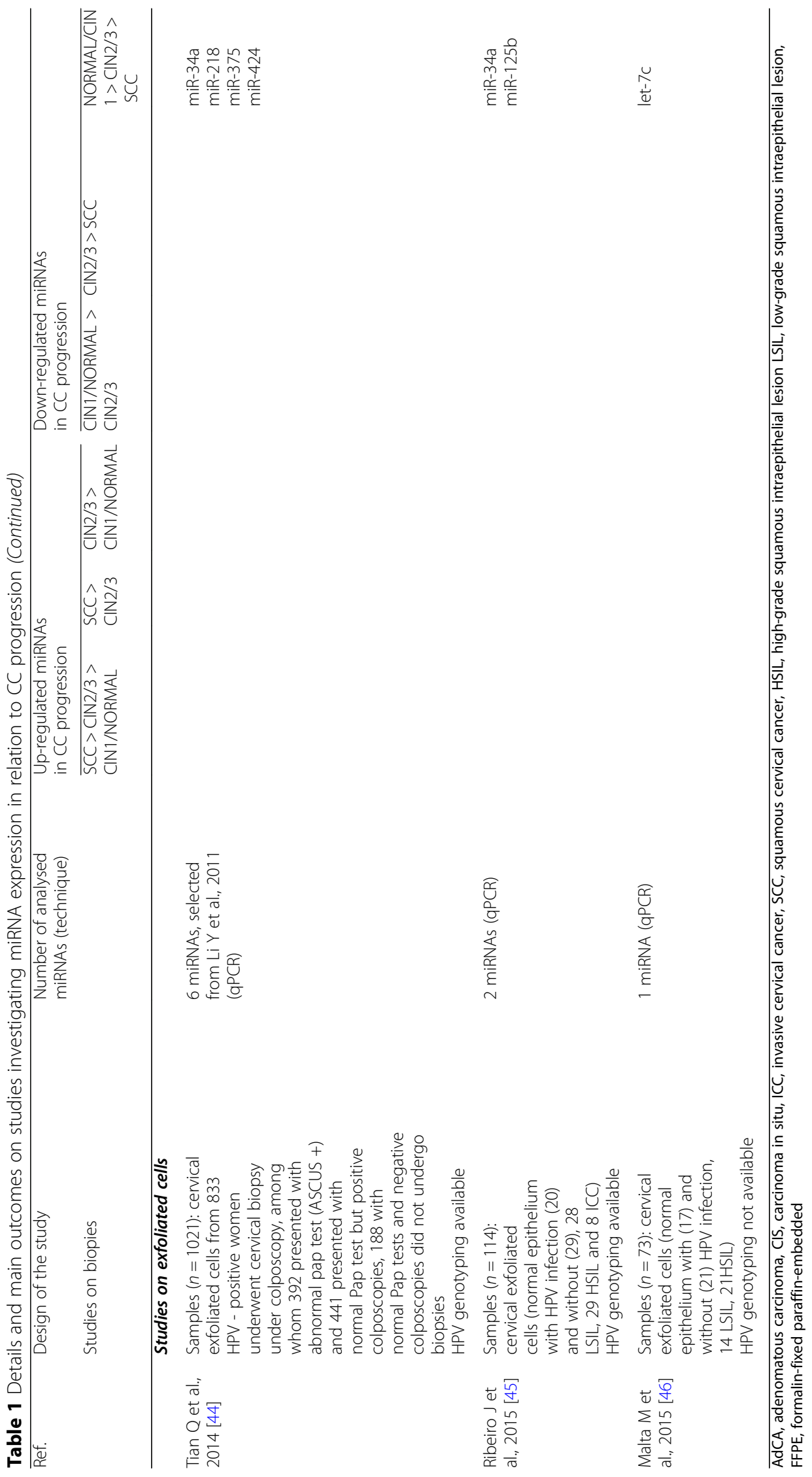


particular, the down-regulation of miR-99a and miR-29a confirmed the results of Pereira et al. [48] just reported above. miR-218 was the most significantly down-regulated, as confirmed by the subsequent studies of Wilting et al. [16], Zeng et al. [50] discussed below and more recently by Jimenez-Vences [41]. In general, miR-218 was under-expressed in tissues infected by High Risk-HPV (HR-HPV) and more in CIN2/3 than in CIN1. In another study by Li Y and colleagues (same first name author, but a different group from that of the array study) [31], this miRNA also presented lower expression levels in patients with CIN2 and CIN3 than in those with CIN1 (78 CIN patients in total). This miRNA has hundreds of target genes, including $L A M B 3$, which has been recognized to increase cell migration and to promote carcinogenesis in mouse models and in human keratinocytes [31]. Li Y and colleagues (of the array study [49]), also compared miRNA expression in CIN2/3 and SCCs with HPV16 infection to normal cervical tissues without HPV infection. The two groups showed significant difference only for miR-375 and miR-99a. Decreasing levels of miR-375, measured by qPCR, with more severe histology were observed also by Bierkens and colleagues on HPV-positive frozen biopsies (6 normal cervical squamous epithelial specimens, 13 CDKN2A-positive HG-CIN, and 9 SCCs) [52].

Finally, in the array study of Li Y and colleagues [49], miR-100 levels were significantly lower in CIN3 than in CIN1 and CIN2, as confirmed subsequently $[16,32]$. This result was also validated in vitro by $\mathrm{Li} \mathrm{BH}$ et al. in cervical cell lines [32]. Down-regulation of miR-100 by its specific inhibitor distinctly promoted cell growth, decreased cell apoptosis, and accelerated G2/M phase progression in $\mathrm{HaCaT}$ cells that constitutively express high level of this miRNA. These findings together suggest that a reduced miR-100 expression may contribute to cervical carcinogenesis by regulating cell growth, cell cycle and apoptosis.

In a study by Cheung and colleagues, the expression of a panel of 202 miRNAs was investigated in normal epithelium, CIN2, CIN3 and ICC samples using a qPCR platform [51]. Twelve miRNAs (10 up- and 2 down-regulated) were differentially expressed in CIN2/3 biopsies compared to normal cervical epithelial cells. This 12-miRNA signature could clearly separate CIN2/3 from normal tissue in an independent group of samples (6 CIN2, 18 CIN3, 9 normal) and patients with SCC (51) from individuals with normal cervical epithelium but not CIN2 from CIN3. Overall, miR-20b and miR-9 showed the highest fold change up-regulation. miR-9 expression was also significantly higher in SCC than in CIN2/3. Authors considered miR-9 of particular interest because it stimulates angiogenesis in a cell-type and context-dependent manner and it is up-regulated in several cancers [51], including ICC [48, 50]. A miR-9-mediated down-regulation of E-cadherin has been shown to lead to activation of $\beta$-catenin, resulting in the up-regulation of the target gene VEGFA, a proangiogenic factor [54]. Down-regulation of E-cadherin has been observed in both CIN and SCC and this is consistent with the progressive up-regulation of miR-9 in CIN and SCC described above [51].

Wilting and colleagues [16] investigated by a microarray the expression of 472 human miRNAs in 47 subjects (normal epithelium, CIN2/3, SCCs) and correlated their differential expression to histology. In total, 106 miRNAs were differentially expressed in CIN2/3 and/or SCCs compared with normal epithelium. Authors identified early transient miRNAs $(n=27$; with significantly different expression in CIN2/3 compared to normal epithelia but with no difference in SCCs vs. normal), late miRNAs ( $n=46$; miRNAs having differential expression in SCCs compared with normal tissue and CIN2/3, but not in CIN2/3 compared with normal tissue), and early continuous miRNAs ( $n=33$; those molecules showing concordant differential expression in SCCs and CIN2/3 compared with normal) (details in Table 1). Some of the altered miRNA expression levels confirmed the results by Pereira et al. [48] and by Cheung et al. [51] (for instance miR-10a up-regulation and miR-203 down-regulation) and by a more recent study of Gocze and colleagues [36]. The latter group also confirmed Wilting's observations of an increase of miR-155 in the transition from CIN1 to SCC, so as Li et al. [16, 49].

More recently, Zeng and colleagues [50] compared miRNA expression profiles in ICC, CIN and normal cervical tissues by microarray analysis and found several miRNAs as significantly dysregulated. In particular, 16 miRNAs were up-regulated and 10 were significantly down-regulated when comparing SCC to normal tissue. miR-21, miR-21-3p (formerly miR-21*), miR-15b and miR-16 were the most over-expressed while miR-218 and miR-376 were the most down-regulated. Forty four miRNAs were differentially expressed (13 up- and 31 down-regulated) when CIN2/3 were compared to normal cervical tissue. No significantly differentially expressed miRNAs were found when comparing CIN1 to normal tissue. Nine miRNAs (miR-21, miR-218, miR-376a, miR-31, miR-630, miR-9, miR-195, miR-497, and miR-199b-5p) differentially expressed in cervical samples with and without lesions were validated by qPCR in 103 samples with similar characteristics. miR-21, miR-31 and miR-9 were again significantly up-regulated in ICC, as found in other works reported in the present review $[16,33,36,37,49,51]$. Among the other validated results, miR-218, miR-195, miR-497 and miR-199b-5p were significantly down-regulated in ICC and CIN2/3, while miR-376a was significantly down-regulated only in ICC but not in CIN2/3. However, they did not observe differential miRNA expression between CIN1 and normal tissue, Authors hypothesized that miRNAs down-regulated in both SCC and CIN2/3 may be involved in the abnormal transformation from pre-cancerous lesions to cancer. 
Table 2 miRNAs up-regulated (A) and down-regulated (B) in CC progression identified in more than one study

\begin{tabular}{|c|c|c|}
\hline References & Number of common miRNAs & miRNAs \\
\hline \multicolumn{3}{|l|}{ A } \\
\hline Li Y et al., 2011 [49]; Wilting et al., 2013 [16] & 5 & $\begin{array}{l}\text { miR-15b }{ }^{a}, \operatorname{miR}-17, \text { miR-92b, } \\
\text { miR-93, miR-185 }\end{array}$ \\
\hline Li Y et al., 2011 [49]; Pereira et al., 2010 [48]; Wilting et al., 2013 [16] & 2 & miR-16, miR-106a \\
\hline Li Y et al., 2011 [49]; Wang et al., 2014 [34]; Wilting et al., 2013 [16] & 2 & miR-92a, miR-25 \\
\hline $\begin{array}{l}\text { Deftereos et al., } 2011 \text { [33]; Shishoida et al., } 2015 \text { [37]; Wilting et al., } \\
2013 \text { [16]; Zeng et al., } 2015 \text { [50]; Bumrungthai et al. } 2015 \text { [40] }\end{array}$ & 1 & miR-21 \\
\hline Cheung et al., 2012 [51]; Pereira et al., 2010 [48]; Wilting et al., 2013 [16] & 1 & miR-10a \\
\hline Cheung et al., 2012 [51]; Li Y et al., 2011 [49]; Wilting et al., 2013 [16] & 1 & miR-20b \\
\hline Gocze et al., 2015 [36]; Li Y et al., 2011 [49]; Wilting et al., 2013 [16] & 1 & miR-155 \\
\hline Cheung et al., 2012 [51]; Wilting et al., 2013 [16]; Zeng et al., 2015 [50] & 1 & miR-9 \\
\hline Li Y et al., 2011 [49]; Zeng et al., 2015 [50]; Zheng et al., 2015 [38] & 1 & miR-31 \\
\hline Gocze et al., 2015 [36]; Pereira et al., 2010 [48] & 1 & miR-27a \\
\hline Wilting et al. 2015 [16]; Ma et al., 2015 [39] & 1 & miR-146a \\
\hline Pereira et al., 2010 [48]; Villegas-Ruiz et al., 2014 [47] & 1 & miR-196a \\
\hline \multicolumn{3}{|l|}{ B } \\
\hline $\begin{array}{l}\text { Li Y et al., } 2011 \text { [49]; Li Y et, } 2010 \text { [31]; Tian et al., } 2014 \text { [44]; Wilting } \\
\text { et al., } 2013 \text { [16]; Zeng et al., } 2015 \text { [50]; Jimenez-Vences et al. } 2016 \text { [41] }\end{array}$ & 1 & miR-218 \\
\hline $\begin{array}{l}\text { Bierkens et al., } 2013 \text { [52]; Li Y et al., } 2011 \text { [49]; Tian et al., } 2014 \text { [44]; } \\
\text { Wilting et al., } 2013 \text { [16] }\end{array}$ & 1 & miR-375 \\
\hline Li Y et al., 2011 [49]; Pereira et al., 2010 [48]; Wilting et al., 2013 [16] & 1 & miR-99a \\
\hline Li Y et al., 2011 [49]; Pereira et al., 2010 [48]; Wang et al., 2014 [34] & 1 & miR-29a \\
\hline $\begin{array}{l}\text { Cheung et al., } 2012 \text { [51]; Pereira et al., } 2010 \text { [48]; Wilting et al., } 2013 \\
\text { [16]; Gocze et al. } 2015 \text { [36] }\end{array}$ & 1 & miR-203 \\
\hline Li Y et al., 2011 [49]; Wilting et al., 2013 [16]; Zeng et al., 2015 [50] & 1 & miR-195 \\
\hline Li Y et al., 2011 [49]; Ribeiro et al., 2015 [45]; Wilting et al., 2013 [16] & 1 & miR-125b \\
\hline Gocze et al., 2015 [36]; Ribeiro et al., 2015 [45]; Tian et al., 2014 [44] & 1 & miR-34a \\
\hline Li Y et al., 2011 [49]; Li. BH et al. 2011 [32]; Wilting et al., 2013 [16] & 1 & miR-100 \\
\hline Li Y et al., 2011 [49]; Pereira et al., 2010 [48] & 1 & miR-145 \\
\hline Li Y et al., 2011 [49]; Tian et al., 2014 [44] & 1 & miR-424 \\
\hline Cheung et al., 2012 [51]; Wilting et al., 2013 [16] & 1 & $\operatorname{miR}-193 b^{b}$ \\
\hline Wilting et al., 2013 [16]; Zeng et al., 2015 [50] & 1 & miR-497 \\
\hline
\end{tabular}

${ }^{a}$ miR-15b is also up-regulated in Wen et al. 2017 [42]

${ }^{b}$ miR-193b is up-regulated in Jimenez-Vences et al. 2016 [41]

Other studies investigated one or few miRNAs previously identified as dysregulated in ICC progression. Villegas-Ruiz and colleagues [47] after an initial investigation of miRNA profiles in tumor vs. healthy tissue by arrays, focused on the expression of miR-196a in relation to ICC progression. They showed increasing expression of this miRNA from healthy tissues to low grade and to high grade CIN and tumor, confirming the findings of Pereira et al. In the study of Gocze [36], miR-196a also showed an increasing expression from CIN1 to CIN2/3 but was significantly lower in SCC than in CIN2/3.

Deftereos et al. [33] analyzed miR-143 and miR-21 in a large set of samples with different stages of disease. Despite increasing down-regulation of miR-143 with increasing severity of histology had been previously reported [47, 48], such pattern was not confirmed in this study [33]. miR-21 expression had been observed to increase with more severe diagnosis in neoplasms of many sites. An overexpressed miR-21 was also found in cervical pre-cancerous lesions and in ICC $[16,36,37,40]$. In the work of Deftereos et al. [33] its levels increased from low-to high-grade CIN and were highest in invasive cancer tissues. miR-21 was also analyzed by Shishoida and colleagues together with let-7a [37]. Increasing miR-21 expression levels were found associated with disease progression while lower levels of let-7a were detected in invasive cancer tissues compared to pre-cancerous lesions and normal control tissues. Among all tissue types, the highest levels of let-7a were detected in 

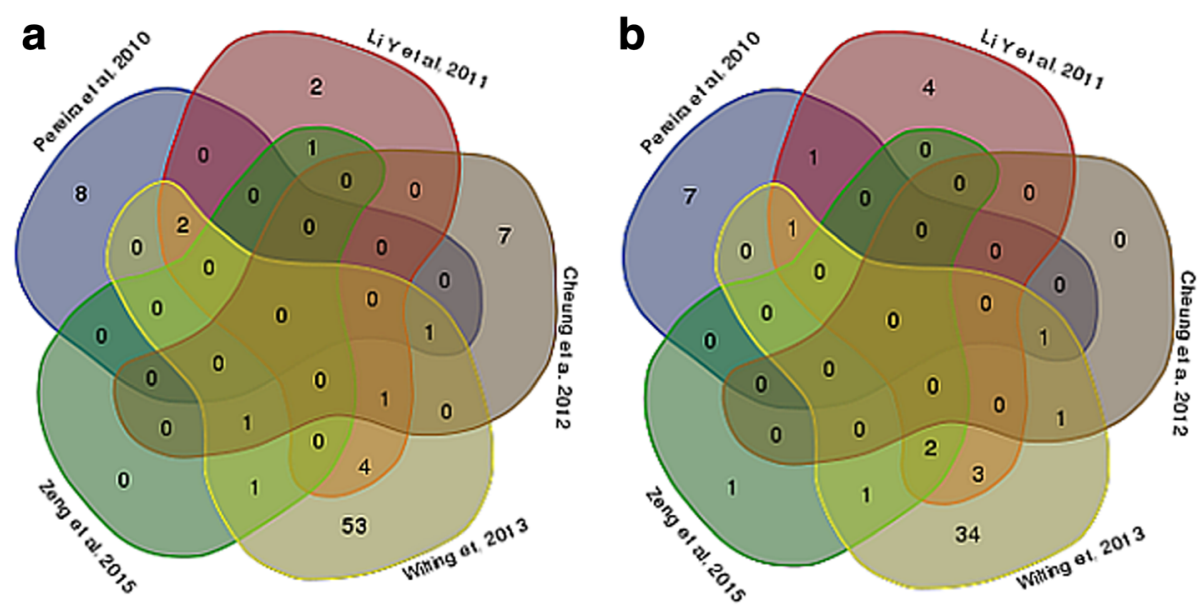

Fig. 1 Venn diagram showing (A) up-regulated and (B) down-regulated miRNAs by studies that used microarrays to identify miRNAs in different stages of CC progression

Disease progression

miR-218, miR-375, miR-99a, miR-29a, miR-203, miR-195, miR-125b,

Down-regulated in multiple studies and with progression miR-34a, miR-100, miR-145, miR-424, miR-193b, miR-497
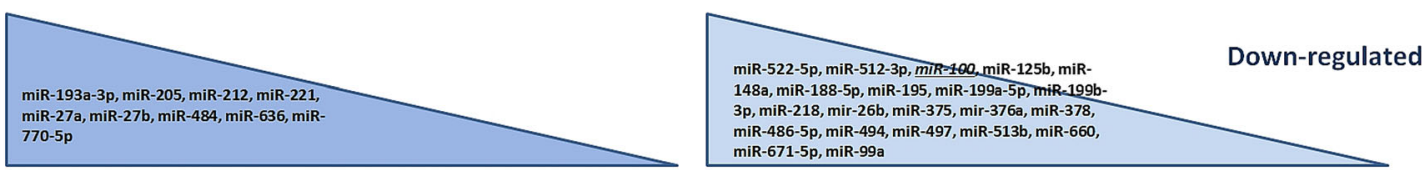
stages
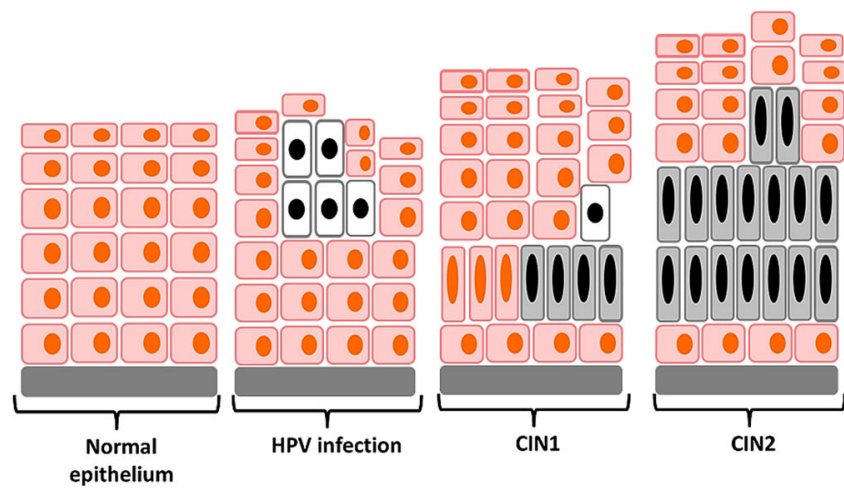

\section{0 \\ IIIIIII}
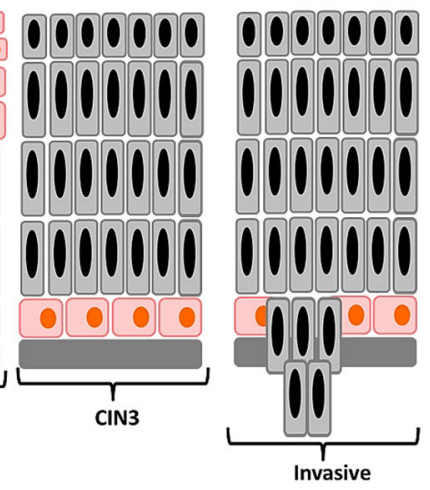

Up-regulated in single stages
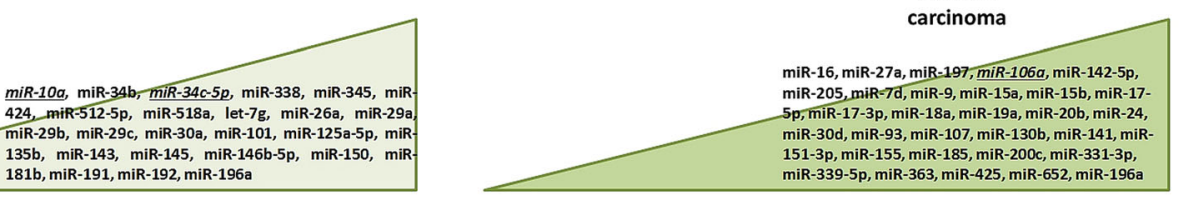

Up-regulated in multiple studies and with progression

Fig. 2 Summary of dysregulated miRNAs in the whole CC progression (miRNAs resulting from multiple studies) and miRNAs associated with progression in intermediate states of CC progression (miRNAs resulting from multiple studies are in Italics and underlined) 
pre-cancerous lesions. Authors observed that, in the cervix, altered miR-21 and let-7a levels were strongly associated with aberrant expression and activation of STAT3 and might be closely linked with HPV16 infection. In this sense, miRNAs could mediate functional links between STAT3 and other transcription factors including, NF-kB. NF-kB pathway is an important player in the development of cervical cancer and with STAT3 cooperatively regulates a number of target genes including antiapoptotic and cell cycle control genes, and genes encoding for cytokines and chemokine [55].

Leung and colleagues observed an overexpression of miR-135a in SCC compared to CIN1, and CIN2/3 in FFPE samples [35]. Authors found that miR-135a regulates Wnt/ $\beta$-catenin signaling through its target genes SIAH1 and possibly $A P C$. This may eventually cause transformation into ICC, with miR-135a also promoting the migration, invasion and proliferation abilities of cancer cells.

In the work of Wang et al. [34], increased levels of miR-25, miR-92a and miR-378 were observed in HPV-infected tissue groups in comparison with HPV negative tissues. These results were consistent with those obtained from HPV-infected raft tissues. Another miRNA, miR-27a, increased its expression from normal cervical tissues to CIN1,2,3 and ICC, in agreement with the study of Gocze et colleagues [36] but at variance with that of Wilting et al. [16], in which miR-27a was down-regulated when moving from normal tissue to ICC. MiR-27a is an oncogenic miRNA modulated by p53, E2F and c-Myc. Both HR-HPV E6 and E7 proteins interact with c-Myc by increasing its transcriptional activity [56]. Finally, in agreement with previous observations [49], miR-29a expression decreased from normal tissue to CIN and ICC.

The study of Li B. et al. [30] examined the expression of pri-miR-34a, the precursor of miR-34a, in normal cervical epithelium, CIN and ICC by semi-quantitative RT-PCR. The pri-miR-34a expression was significantly decreased in the CIN and ICC groups in comparison with normal cervical epithelium group as a function of grade. The reduction of pri-miR-34a expression associated with HR-HPV infection occurred before morphologic abnormalities of cervical epithelium and before the changes induced by HR-HPV E6 in the p53-dependent pathway. Thus, authors concluded that it is plausibly an early-onset event in ICC development and that miR-34a and its precursor can likely be regarded as potential molecular markers for cervical screening and molecular targets for blocking ICC development.

\section{miRNA alterations in ICC progression analyzed in exfoliated cells}

Cervical exfoliated cells are widely used in ICC screening, both for HPV testing and Pap test. Recently, their use has been extended to miRNA analyses. To our knowledge, the first report on miRNA detection in cervical exfoliated cells was by Tian et al. in 2014 [44]. Six candidate miRNAs, selected among those identified by Li Y and colleagues [49], were investigated in samples from $1021 \mathrm{HPV}$-positive women. The levels of miR-218, miR-34a, miR-424 and miR-375 significantly decreased with increasing severity.

Results partially confirm those previously obtained in cervical tissue. In particular, a decrease in the levels of miR-424 and miR-375 with higher-grade cytology was observed, similar to that seen when moving from CIN1 to CIN3 tissues [49].

Ribeiro et al. [45] evaluated miR-34a and miR-125b levels in cervical exfoliated cells of women with different histology (normal epithelium with and without HPV infection, Low-grade CIN, High-grade CIN and ICC). The study revealed an increased expression of miR-125b among women with HPV infection but normal epithelium and a significant progressive down-regulation with increasing severity of cervical lesions, reaching an $80 \%$ reduction in ICC. Women with normal cervix and HPV infection had also increased miR-34a expression levels. Despite no significant correlation with severity of cervical lesions, its expression increased in ICC. Significantly lower levels of miR-34a were detected also in CIN2/3 when compared with CIN1 and in SCC when compared with CIN2/3 (similarly to what observed in tissues by Gocze et al. [36]). It has been observed that miR-34a is directly regulated by p53 and that HR-HPV E6 induces its inhibition through p53 [36]. These observations confirm that miR-34a acts as a tumor suppressive miRNA in HPV-induced cervical transformation. This alteration in miR-34a expression is associated with the presence of a single or of multiple HR-HPV types.

let-7c levels were also evaluated by qPCR in cervical exfoliated cells from women with normal epithelium or with CIN (but no ICC) in the study by Malta and colleagues [46]. let-7c was progressively down-regulated in CIN of increasing grade. No previous investigations characterized let-7c expression during the progression of cervical lesions to cancer. let-7c has been reported as a probable target of p53 and its expression is induced in response to p53 activation [46].

These few studies shows that the potential application of miRNA detection in cervical exfoliated cells deserves further exploration, also as an additional option for triage of HPV-positive women in population-based screening.

Commonly identified dysregulated miRNAs: their overlap among studies and target enrichment

The list of miRNAs found to be up- (17) or down- (13) regulated in relation to ICC progression and repeatedly observed among studies is reported in Table 2 and Additional file 2: Table S1. Among over expressed 
miRNAs, one (miR-21) was found to be associated to cervical carcinogenesis by five studies, nine (miR-9, miR-16, miR-25, miR-10a, miR-20b, miR-31 miR-92a, miR-106a and miR-155) by 3 studies and eight (miR-15b, miR-17, miR-27a, miR-92b, miR-93, miR-146a, miR-185 and miR-196a) by 2 studies. Among under-expressed miRNAs, for one (miR-218) an association was found by six studies, for two (miR-375 and miR-203) by four studies and for six (miR-99a, miR-29a, miR-195, miR-125b, miR-34a and miR-100) by three studies each. Fig. 1 reports the overlap between array-based studies, which was in fact very small.

The number of experimentally validated target genes for all the above mentioned miRNAs, retrieved in the miRWalk 2.0 database, is reported in Additional file 2: Table S2. Some 16,671 target genes were identified for the up-regulated miRNAs and 7075 for those down-regulated. After removing multiple miRNA binding sites for each target gene, genes overlapping within each group of miRNAs and those in common between up-regulated and down-regulated miRNAs, 3395 and 1575 genes, respectively, remained. Gene enrichment analysis for the final list of target genes of down-regulated miRNAs provided statistically significant over-representation and biologically plausible Kegg pathways terms (Additional file 2: Table S3). Subsequently, the enrichment analysis was focused only on the genes that were targeted by miRNAs found up-regulated in more studies (Additional file 2: Table S4). In this case, an overrepresentation of Kegg pathway terms (Additional file 2: Table S5) and Virus Mint terms emerged (Additional file 2: Table S6).

Finally, both original lists of miRNA validated target genes were investigated also in the CCDB database, where 257 genes over- and 110 under-expressed in ICC are listed. Among those genes previously identified as targets for down-regulated miRNAs (thus expected to be over-expressed with progression to ICC), 22 (8.5\%) were indeed reported as over-expressed in ICC in CCDB. On the other hand, among the target genes of up-regulated miRNAs (thus expected to be under-expressed with increasing severity), 34 (30.9\%) were also among the genes listed as under-expressed in ICC (Additional file 2: Table S7). Enrichment analyses have been performed on these resulting genes (Additional file 2: Tables S8, S9).

\section{Discussion}

In summary, the expression levels of several miRNAs were repeatedly found to be associated to progression towards ICC via pre-cancerous lesions in different studies (Fig. 2). Interestingly, for many of them deregulation was not only confirmed by an internal validation in the same study but also with other techniques by other researchers. For instance, miR29a was found to be down-regulated with ICC progression [49] both when its expression levels were analyzed by an array-approach or tested by qPCR in an independent set of samples. Similar observations were obtained by Wang and colleagues [34], who first investigated the same miRNA by array and validated such results by qPCR on a large sample set.

Among up-regulated miRNAs, miR-21, which was found associated to CIN and ICC in 4 studies [16, 33, $37,50]$, is of particular interest. miR-21 is one of the most abundantly expressed miRNA in mammals. Its up-regulation is associated with many cancers, both derived from solid tissues and of leukemic origin [57]. The identification of miR-21 function was the object of many research groups in the last 10 years. miR-21 interacts with a large number of targets and it is finely regulated in response to extracellular signals. Although miR-21 has been recognized as a key regulator of many processes involved in the control of cell survival and proliferation, it has also been linked to key processes in inflammation. Unlike other molecules, miR-21 is not simply characteristic of a pro-inflammatory or immunosuppressive status, but it acts as a key signal mediating their balance $[57,58]$. Other miRNAs found altered in the present review, such as miR-125b, miR-155, and miR-146a, are known to be important in immune response and inflammation [59]. As an example, miR-125b is repeatedly observed down-regulated in relation to ICC progression. Interestingly, this miRNA has an important role in immune response and inflammation but can act as both an oncogene and oncosuppressor. miR-125b seems to be associated with HPV-induced carcinogenesis in two distinct pathways: a) it has homology for $H P V-L 2$, which is needed for the viral capsid assembly (so it is possible that after early infection miR-125b can inhibit HPV replication and improve viral clearance); and b) miR-125b leads to p53-pathway inactivation, thus maintaining cells viable with viral genomes inside. This may result in the observed increased risk of HPV-genome integration and neoplastic lesion development [20]. To discern between an involvement of miRNAs in inflammatory processes related to HPV infection and their role in malignant transformation remains still an open issue, which needs to be furtherly explored [40].

Among down-regulated miRNAs, miR-34a, miR-125 and miR-375 were found to be progressively dysregulated when moving from normal epithelium to ICC, both in tissues and in exfoliated cells. This finding opens the possibility of an application in the screening process, to be tested in larger populations. From an epidemiological point of view, the main limitation of existing studies is the limited number of subjects included and, even more, in their being possibly selected. In principle, only samples including all lesions detected in a population-based screening and a random sample of healthy women from the same population (or of HPV-positive women without lesions if the conclusions are to be applied to 
HPV-positive women) are not subject to selection bias. Most studies seem, instead, to have been conducted on convenience material, whose origin and possible selection is usually not discussed in detail.

Some miRNAs were dysregulated in all stages of carcinogenesis, others only when moving from CIN2/3 to ICC $[16,36,48,51]$ (Fig. 2). In one study, miR-196 increased from normal epithelium to CIN3 and then decreases from CIN3 to ICC [36].

When investigating the role of the experimentally validated target genes of the miRNAs identified as dysregulated in cervical carcinogenesis, we found many pathways relevant to ICC onset and progression, such as signaling pathways. Interestingly, the overlap between the results of microarray-based studies was limited. This may be due to differences in study designs and populations (i.e. different ethnicities) or to the small number of ICC and CIN investigated. However, we must also report that all studies used different arrays, thus containing different number and type of miRNA probes or based on different array technology. The most interesting miRNAs found as dysregulated in multiple studies include miR-10a $[16,48,51]$, miR-20b [16, 49, 51], miR-9 [16, 50, 51], miR-16 and miR-106a [16, 48, 49] (all up-regulated) and miR-99a [16, 48, 49], miR-203 $[16,48,51]$, and miR-195 [16, 49, 50] (down-regulated).

Array-based analysis is restricted to miRNA molecules provided by databases and suffers from cross-detection-prone hybridization methods. As we could notice in the present review, despite the rapidly increasing number of miRNAs discovered and registered (currently 2656 human mature miRNAs; http://www.mirbase.org/ index.shtml, Release 22 March 2018) the majority of studies focused on the first 800 miRNAs. Recently the use of next-generation sequencing (NGS) technology for miRNA expression profiling increased [60]. NGS offers a genome-wide approach and allows overcoming cross hybridization problems. Small RNA-sequencing, allowing also the detection of other similar small noncoding RNAs (such as piRNAs and tRNAs), has the greatest detection sensitivity and the highest accuracy in differential expression analysis. For the application of NGS techniques to small RNAs the standardization of experimental and computational protocols just begun [61]. Nevertheless, an important increase of studies applying NGS to miRNAs, also in cervical carcinogenesis, is expected in the next future [62]. The ideal approach would be to apply NGS on homogenous large scale studies for an hypothesis-free investigation of the whole miRNome to detect robust potential candidate (s) miRNA relevant for ICC progression as well as high-risk HPV infection. This is to avoid conflicting outcomes from small studies analyzing restricted lists of candidate miRNAs. Finally, small specific miRNA signatures should be evicted to be applied on a very extended scale, also in countries with more limited availability of funds for molecular analyses in the clinical routine. The first molecular characterization, including miRNAs, of 228 primary cervical cancers in comparison with healthy tissues has been recently described by The Cancer Genome Atlas (TCGA) network [63].

\section{Conclusions}

In the context of ICC screening, an important issue to be investigated is whether miRNA signatures could be associated with current and future risk of HG-CIN, most likely in combination with other different markers, in order to improve risk stratification. Some of these markers could be used to identify very recently arisen HG-CIN. As the latter have very low probability of progression to invasion in short time and high probability of regression, this would allow a "wait and see" management, resulting in reduced overtreatment. The investigation of miRNA levels in cervical exfoliated cells certainly opens new possibilities for studying molecular markers in the context of screening programs.

\section{Additional files}

\begin{abstract}
Additional file 1: Figure S1. Workflow of selection of the studies included in the present Review. (TIF 1519 kb)

Additional file 2: Table S1. Dysregulated miRNAs in ICC progression in multiple studies as retrieved from the reviewed literature. Table S2. Validated target genes for the dysregulated miRNAs in ICC progression in multiple studies (data retrieved from miRWalk2.0). Table S3. Enrichment analysis for validated target genes of miRNA down-regulated in ICC progression (Kegg Pathways). Table S4. A group of 5 up-regulated miRNAs retrieved by multiple studies in the present review. Table S5. Enrichment analyses for genes targeted $(n=2620)$ by the above 5 miRNAs up-regulated in ICC progression (Kegg Pathways). Table S6. Enrichment analyses for genes targeted $(n=2620)$ by the above 5 miRNAs up-regulated in ICC progression (Virus Mint). Table S7. Altered expression levels of genes relevant for ICC (reported in CCDB) targeted by dysregulated miRNAs in ICC progression. (DOCX $39 \mathrm{~kb}$ )
\end{abstract}

\section{Acknowledgments}

Authors would like to express their gratitude to Mr. Eric Van Emburgh for his valuable contribution to the preparation of the manuscript.

\section{Funding}

Study supported by Italian Association on Cancer Research (AIRC, IG2013 N.14119 to GR), European Union (CoHeahr project, FP7 grant agreement n HEALTH-F3-2013e603019 to GR), by Fondazione Umberto Veronesi "Post-doctoral fellowship" (to BP), and by Compagnia di San Paolo (AN, DDM, AF, (DG).

The funding bodies had no role in the design of the study and collection, analysis, and interpretation of data and in writing the manuscript. However, the funding bodies sustained the research of the authors AN, DDM, AF and BP for their salaries.

Consent to publish

Not applicable to this study.

\section{Authors' contributions}

Conception and design of the study: BP, AN, GR. Acquisition, analysis and interpretation of data: $B P, A N, D D M, G R, A F, C D G$. Draft of the manuscript: BP, AN, DDM, AF. Critical revision of the manuscript: GR, CDG All coauthors have read the manuscript and approved the final version for submission and publication to this journal. 


\section{Ethics approval and consent to participate}

Not applicable to this study.

\section{Competing interests}

The authors declare that they do not have any competing interest.

\section{Publisher's Note}

Springer Nature remains neutral with regard to jurisdictional claims in published maps and institutional affiliations.

\section{Author details}

${ }^{1}$ Italian Institute for Genomic Medicine (IIGM), Via Nizza 52, 10126 Turin, Italy. ${ }^{2}$ Department of Medical Sciences, University of Turin, Via Santena 19, 10126 Turin, Italy. ${ }^{3}$ Center for Cancer Epidemiology and Prevention, AO City of Health and Science, Via Cavour, 3110123 Turin, Italy.

\section{Received: 8 August 2017 Accepted: 13 June 2018}

\section{Published online: 27 June 2018}

\section{References}

1. Ferlay J, Soerjomataram I, Dikshit R, Eser S, Mathers C, Rebelo M, Parkin DM, Forman D, Bray F. Cancer incidence and mortality worldwide: sources, methods and major patterns in GLOBOCAN 2012. Int J Cancer. 2015;136(5): E359-86.

2. Bosch FX, Broker TR, Forman D, Moscicki AB, Gillison ML, Doorbar J, Stern PL, Stanley M, Arbyn M, Poljak M, et al. Comprehensive control of human papillomavirus infections and related diseases. Vaccine. 2013;31(Suppl 7):H1-31.

3. Rodriguez AC, Schiffman M, Herrero R, Wacholder S, Hildesheim A, Castle PE, Solomon D, Burk R. Rapid clearance of human papillomavirus and implications for clinical focus on persistent infections. J Natl Cancer Inst. 2008;100(7):513-7.

4. McCredie MR, Paul C, Sharples KJ, Baranyai J, Medley G, Skegg DC, Jones RW. Consequences in women of participating in a study of the natural history of cervical intraepithelial neoplasia 3. Aust N Z J Obstet Gynaecol. 2010:50(4):363-70

5. Castle PE, Schiffman M, Wheeler CM, Solomon D. Evidence for frequent regression of cervical intraepithelial neoplasia-grade 2. Obstet Gynecol. 2009;113(1):18-25

6. Steenbergen RD, Snijders PJ, Heideman DA, Meijer CJ. Clinical implications of (epi) genetic changes in HPV-induced cervical precancerous lesions. Nat Rev Cancer. 2014;14(6):395-405.

7. Schiffman M, Yu K, Zuna R, Terence Dunn S, Zhang H, Walker J, Gold M, Hyun N, Rydzak G, Katki HA, et al. Proof-of-principle study of a novel cervical screening and triage strategy: computer-analyzed cytology to decide which HPV-positive women are likely to have >/=CIN2. Int J Cancer. 2017;140(3): $718-25$

8. Ronco G, Dillner J, Elfstrom KM, Tunesi S, Snijders PJ, Arbyn M, Kitchener H Segnan N, Gilham C, Giorgi-Rossi P, et al. Efficacy of HPV-based screening for prevention of invasive cervical cancer: follow-up of four European randomised controlled trials. Lancet. 2014;383(9916):524-32.

9. Ronco G, Zappa M, Franceschi S, Tunesi S, Caprioglio A, Confortini M, Del Mistro A, Carozzi F, Segnan N, Zorzi M, et al. Impact of variations in triage cytology interpretation on human papillomavirus-based cervical screening and implications for screening algorithms. Eur J Cancer. 2016;68:148-55.

10. Farazi TA, Hoell Jl, Morozov P, Tuschl T. MicroRNAs in human cancer. Adv Exp Med Biol. 2013;774:1-20.

11. Palanichamy JK, Rao DS. miRNA dysregulation in cancer: towards a mechanistic understanding. Front Genet. 2014;5:54

12. Macfarlane LA, Murphy PR. MicroRNA: biogenesis, function and role in Cancer. Curr Genomics. 2010;11(7):537-61.

13. Lin S, Gregory RI. MicroRNA biogenesis pathways in cancer. Nat Rev Cancer. 2015;15(6):321-33

14. Davis-Dusenbery BN, Hata A. MicroRNA in Cancer: the involvement of aberrant MicroRNA biogenesis regulatory pathways. Genes Cancer. 2010; 1(11):1100-14

15. Iorio MV, Croce CM. Causes and consequences of microRNA dysregulation Cancer J. 2012;18(3):215-22.

16. Wilting SM, Snijders PJ, Verlaat W, Jaspers A, van de Wiel MA, van Wieringen WN, Meijer GA, Kenter GG, Yi Y, le Sage C, et al. Altered microRNA expression associated with chromosomal changes contributes to cervical carcinogenesis. Oncogene. 2013;32(1):106-16.
17. Rao Q, Shen Q, Zhou H, Peng Y, Li J, Lin Z. Aberrant microRNA expression in human cervical carcinomas. Med Oncol. 2012;29(2):1242-8.

18. Zheng ZM, Wang X. Regulation of cellular miRNA expression by human papillomaviruses. Biochim Biophys Acta. 2011;1809(11-12):668-77.

19. Pedroza-Torres A, Lopez-Urrutia E, Garcia-Castillo V, Jacobo-Herrera N, Herrera LA, Peralta-Zaragoza O, Lopez-Camarillo C, De Leon DC, FernandezRetana J, Cerna-Cortes JF, et al. MicroRNAs in cervical cancer: evidences for a miRNA profile deregulated by HPV and its impact on radio-resistance. Molecules. 2014;19(5):6263-81.

20. Ribeiro J, Sousa H. MicroRNAs as biomarkers of cervical cancer development: a literature review on miR-125b and miR-34a. Mol Biol Rep. 2014;41(3):1525-31.

21. Sharma G, Dua P, Agarwal SM. A comprehensive review of dysregulated miRNAs involved in cervical Cancer. Curr Genomics. 2014;15(4):310-23.

22. Wilting $S M$, Steenbergen $R D$, Tijssen $M$, van Wieringen $W N$, Helmerhorst $T J$, van Kemenade FJ, Bleeker MC, van de Wiel MA, Carvalho B, Meijer GA, et al. Chromosomal signatures of a subset of high-grade premalignant cervical lesions closely resemble invasive carcinomas. Cancer Res. 2009;69(2):647-55.

23. Chaiwongkot A, Vinokurova S, Pientong C, Ekalaksananan T, Kongyingyoes B, Kleebkaow P, Chumworathayi B, Patarapadungkit N, Reuschenbach M, von Knebel Doeberitz M. Differential methylation of E2 binding sites in episomal and integrated HPV 16 genomes in preinvasive and invasive cervical lesions. Int J Cancer. 2013;132(9):2087-94.

24. He Y, Lin J, Ding Y, Liu G, Luo Y, Huang M, Xu C, Kim TK, Etheridge A, Lin M, et al. A systematic study on dysregulated microRNAs in cervical cancer development. Int J Cancer. 2016;138(6):1312-27.

25. Shishodia G, Verma G, Das BC, Bharti AC. miRNA as viral transcription tuners in HPV-mediated cervical carcinogenesis. Front Biosci (Schol Ed). 2018;10:21-47.

26. Gonzalez-Quintana V, Palma-Berre L, Campos-Parra AD, Lopez-Urrutia E, Peralta-Zaragoza O, Vazquez-Romo R, Perez-Plasencia C. MicroRNAs are involved in cervical cancer development, progression, clinical outcome and improvement treatment response (review). Oncol Rep. 2016:35(1):3-12.

27. Dweep H, Sticht C, Pandey P, Gretz N. miRWalk-database: prediction of possible miRNA binding sites by "walking" the genes of three genomes. J Biomed Inform. 2011;44(5):839-47.

28. Chen EY, Tan CM, Kou Y, Duan Q, Wang Z, Meirelles GV, Clark NR, Ma'ayan A. Enrichr: interactive and collaborative HTML5 gene list enrichment analysis tool. BMC Bioinformatics. 2013:14:128.

29. Agarwal SM, Raghav D, Singh H, Raghava GP. CCDB: a curated database of genes involved in cervix cancer. Nucleic Acids Res. 2011:39(Database):D975-9.

30. Li B, Hu Y, Ye F, Li Y, Lv W, Xie X. Reduced miR-34a expression in normal cervical tissues and cervical lesions with high-risk human papillomavirus infection. International journal of gynecological cancer : official journal of the International Gynecological Cancer Society. 2010;20(4):597-604.

31. Li Y, Liu J, Yuan C, Cui B, Zou X, Qiao Y. High-risk human papillomavirus reduces the expression of microRNA-218 in women with cervical intraepithelial neoplasia. The Journal of international medical research. 2010; 38(5):1730-6.

32. Li BH, Zhou JS, Ye F, Cheng XD, Zhou CY, Lu WG, Xie X. Reduced miR-100 expression in cervical cancer and precursors and its carcinogenic effect through targeting PLK1 protein. Eur J Cancer. 2011;47(14):2166-74.

33. Deftereos G, Corrie SR, Feng Q, Morihara J, Stern J, Hawes SE, Kiviat NB. Expression of mir-21 and mir-143 in cervical specimens ranging from histologically normal through to invasive cervical cancer. PLoS One. 2011; 6(12):e28423.

34. Wang X, Wang HK, Li Y, Hafner M, Banerjee NS, Tang S, Briskin D, Meyers C, Chow LT, Xie $X$, et al. microRNAs are biomarkers of oncogenic human papillomavirus infections. P Natl Acad Sci USA. 2014;111(11):4262-7.

35. Leung CO, Deng W, Ye TM, Ngan HY, Tsao SW, Cheung AN, Pang RT, Yeung WS. miR-135a leads to cervical cancer cell transformation through regulation of beta-catenin via a SIAH1-dependent ubiquitin proteosomal pathway. Carcinogenesis. 2014;35(9):1931-40.

36. Gocze K, Gombos K, Kovacs K, Juhasz K, Gocze P, Kiss I. MicroRNA expressions in HPV-induced cervical dysplasia and cancer. Anticancer Res. 2015;35(1):523-30

37. Shishodia G, Shukla S, Srivastava Y, Masaldan S, Mehta S, Bhambhani S, Sharma S, Mehrotra R, Das BC, Bharti AC. Alterations in microRNAs miR-21 and let-7a correlate with aberrant STAT3 signaling and downstream effects during cervical carcinogenesis. Mol Cancer. 2015;14:116.

38. Zheng W, Liu Z, Zhang W, Hu X. miR-31 functions as an oncogene in cervical cancer. Arch Gynecol Obstet. 2015;292(5):1083-9. 
39. Ma L, Hong Y, Lu C, Chen Y, Ma C. The occurrence of cervical cancer in Uygur women in Xinjiang Uygur autonomous region is correlated to microRNA-146a and ethnic factor. Int J Clin Exp Pathol. 2015;8(8):9368-75.

40. Bumrungthai $S$, Ekalaksananan T, Evans MF, Chopjitt $P$, Tangsiriwatthana $T$, Patarapadungkit N, Kleebkaow P, Luanratanakorn S, Kongyingyoes B, Worawichawong $S$, et al. Up-regulation of miR-21 is associated with cervicitis and human papillomavirus infection in cervical tissues. PLoS One. 2015;10(5):e0127109

41. Jimenez-Wences H, Martinez-Carrillo DN, Peralta-Zaragoza O, Campos-Viguri GE, Hernandez-Sotelo D, Jimenez-Lopez MA, Munoz-Camacho JG, GarzonBarrientos VH, Illades-Aguiar B, Fernandez-Tilapa G. Methylation and expression of miRNAs in precancerous lesions and cervical cancer with HPV16 infection. Oncol Rep. 2016;35(4):2297-305.

42. Wen F, Xu JZ, Wang XR. Increased expression of miR-15b is associated with clinicopathological features and poor prognosis in cervical carcinoma. Arch Gynecol Obstet. 2017;295(3):743-9.

43. Sun P, Shen Y, Gong JM, Zhou LL, Sheng JH, Duan FJ, New A. MicroRNA expression signature for cervical Cancer. International journal of gynecological cancer : official journal of the International Gynecological Cancer Society. 2017;27(2):339-43

44. Tian Q, Li Y, Wang F, Xu J, Shen Y, Ye F, Wang X, Cheng X, Chen Y, Wan X, et al. MicroRNA detection in cervical exfoliated cells as a triage for human papillomavirus-positive women. J Natl Cancer Inst. 2014;106(9):dju241.

45. Ribeiro J, Marinho-Dias J, Monteiro P, Loureiro J, Baldaque I, Medeiros R, Sousa H. miR-34a and miR-125b expression in HPV infection and cervical Cancer development. Biomed Res Int. 2015;2015:304584.

46. Malta M, Ribeiro J, Monteiro P, Loureiro J, Medeiros R, Sousa H. Let-7c is a candidate biomarker for cervical intraepithelial lesions: a pilot study. Molecular diagnosis \& therapy. 2015;19(3):191-6.

47. Villegas-Ruiz V, Juarez-Mendez S, Perez-Gonzalez OA, Arreola H, PaniaguaGarcia L, Parra-Melquiadez M, Peralta-Rodriguez R, Lopez-Romero R, Monroy-Garcia A, Mantilla-Morales A, et al. Heterogeneity of microRNAs expression in cervical cancer cells: over-expression of miR-196a. Int J Clin Exp Pathol. 2014:7(4):1389-401.

48. Pereira PM, Marques JP, Soares AR, Carreto L, Santos MA. MicroRNA expression variability in human cervical tissues. PLoS One. 2010:5(7):e11780.

49. Li Y, Wang F, Xu J, Ye F, Shen Y, Zhou J, Lu W, Wan X, Ma D, Xie X. Progressive miRNA expression profiles in cervical carcinogenesis and identification of HPVrelated target genes for miR-29. J Pathol. 2011;224(4):484-95.

50. Zeng K, Zheng W, Mo X, Liu F, Li M, Liu Z, Zhang W, Hu X. Dysregulated microRNAs involved in the progression of cervical neoplasm. Arch Gyneco Obstet. 2015;292(4):905-13.

51. Cheung TH, Man KN, Yu MY, Yim SF, Siu NS, Lo KW, Doran G, Wong RR, Wang WW, Smith DI, et al. Dysregulated microRNAs in the pathogenesis and progression of cervical neoplasm. Cell Cycle. 2012;11(15):2876-84.

52. Bierkens M, Krijgsman O, Wilting SM, Bosch L, Jaspers A, Meijer GA, Meijer CJ, Snijders PJ, Ylstra B, Steenbergen RD. Focal aberrations indicate EYA2 and hsa-miR-375 as oncogene and tumor suppressor in cervical carcinogenesis. Genes, chromosomes \& cancer. 2013:52(1):56-68.

53. Bustin SA, Benes V, Garson JA, Hellemans J, Huggett J, Kubista M, Mueller R, Nolan T, Pfaffl MW, Shipley GL, et al. The MIQE guidelines: minimum information for publication of quantitative real-time PCR experiments. Clin Chem. 2009:55(4):611-22.

54. Ma L, Young J, Prabhala H, Pan E, Mestdagh P, Muth D, Teruya-Feldstein J, Reinhardt F, Onder TT, Valastyan S, et al. miR-9, a MYC/MYCN-activated microRNA, regulates E-cadherin and cancer metastasis. Nat Cell Biol. 2010; 12(3):247-56.

55. Tilborghs S, Corthouts J, Verhoeven Y, Arias D, Rolfo C, Trinh XB, van Dam PA. The role of nuclear factor-kappa $B$ signaling in human cervical cancer. Crit Rev Oncol Hematol. 2017;120:141-50

56. Wang YW, Chang HS, Lin CH, Yu WC. HPV-18 E7 conjugates to c-Myc and mediates its transcriptional activity. Int J Biochem Cell Biol. 2007;39(2):402-12

57. Sheedy FJ. Turning 21: induction of miR-21 as a key switch in the inflammatory response. Front Immunol. 2015;6:19.

58. Huang Y, He Y, Li J. MicroRNA-21: a central regulator of fibrotic diseases via various targets. Curr Pharm Des. 2015;21(17):2236-42.

59. Mangino G, Chiantore MV, Iuliano M, Fiorucci G, Romeo G. Inflammatory microenvironment and human papillomavirus-induced carcinogenesis. Cytokine Growth Factor Rev. 2016;30:103-11.
60. Stokowy T, Eszlinger M, Swierniak M, Fujarewicz K, Jarzab B, Paschke R, Krohn K. Analysis options for high-throughput sequencing in miRNA expression profiling. BMC Res Notes. 2014;7:144.

61. Buschmann D, Haberberger A, Kirchner B, Spornraft M, Riedmaier I, Schelling G, Pfaffl MW. Toward reliable biomarker signatures in the age of liquid biopsies - how to standardize the small RNA-Seq workflow. Nucleic Acids Res. 2016:44(13):5995-6018.

62. Lopez JP, Diallo A, Cruceanu C, Fiori LM, Laboissiere S, Guillet I, Fontaine J, Ragoussis J, Benes V. Turecki G, et al. Biomarker discovery: quantification of microRNAs and other small non-coding RNAs using next generation sequencing. BMC Med Genet. 2015:8:35.

63. Integrated genomic and molecular characterization of cervical cancer. Nature. 2017:543(7645):378-84

\section{Ready to submit your research? Choose BMC and benefit from:}

- fast, convenient online submission

- thorough peer review by experienced researchers in your field

- rapid publication on acceptance

- support for research data, including large and complex data types

- gold Open Access which fosters wider collaboration and increased citations

- maximum visibility for your research: over $100 \mathrm{M}$ website views per year

At BMC, research is always in progress.

Learn more biomedcentral.com/submissions 